\title{
Why it pays to conceal - on the optimal timing of acquiring verifiable information
}

Citation for published version (APA):

Feess, E., \& Walzl, M. (2006). Why it pays to conceal - on the optimal timing of acquiring verifiable information. METEOR, Maastricht University School of Business and Economics. METEOR Research Memorandum No. 020 https://doi.org/10.26481/umamet.2006020

Document status and date:

Published: 01/01/2006

DOI:

10.26481/umamet.2006020

Document Version:

Publisher's PDF, also known as Version of record

\section{Please check the document version of this publication:}

- A submitted manuscript is the version of the article upon submission and before peer-review. There can be important differences between the submitted version and the official published version of record.

People interested in the research are advised to contact the author for the final version of the publication, or visit the DOI to the publisher's website.

- The final author version and the galley proof are versions of the publication after peer review.

- The final published version features the final layout of the paper including the volume, issue and page numbers.

Link to publication

\footnotetext{
General rights rights.

- You may freely distribute the URL identifying the publication in the public portal. please follow below link for the End User Agreement:

www.umlib.nl/taverne-license

Take down policy

If you believe that this document breaches copyright please contact us at:

repository@maastrichtuniversity.nl

providing details and we will investigate your claim.
}

Copyright and moral rights for the publications made accessible in the public portal are retained by the authors and/or other copyright owners and it is a condition of accessing publications that users recognise and abide by the legal requirements associated with these

- Users may download and print one copy of any publication from the public portal for the purpose of private study or research.

- You may not further distribute the material or use it for any profit-making activity or commercial gain

If the publication is distributed under the terms of Article $25 \mathrm{fa}$ of the Dutch Copyright Act, indicated by the "Taverne" license above, 
Eberhard Feess, Markus Walzl

Why it Pays to Conceal - On the Optimal Timing of Acquiring Verifiable Information

RM/06/020

JEL code: D02, D82, D86

\section{METE@R}

Maastricht research school of Economics of TEchnology and ORganizations

Universiteit Maastricht

Faculty of Economics and Business Administration

P.O. Box 616

NL - 6200 MD Maastricht

phone : :+31 433883830

fax $\quad$ : ++31433884873 



\title{
Why it Pays to Conceal - On the Optimal Timing of Acquiring Verifiable Information*
}

\author{
Eberhard Feess ${ }^{\dagger}$ and Markus Walzl
}

May 31, 2006

\begin{abstract}
We consider optimal contracts when a principal has two sources to detect bad projects. The first one is an information technology without agency costs $\left(I T_{P}\right)$, whereas the second one is the expertise of an agent subject to moral hazard, adverse selection and limited liability $\left(I T_{A}\right)$. First, we show that the principal does not necessarily benefit from access to additional information and thereby may prefer to ignore it. Second, we discuss different timings of information release, i.e. a disclosure contract offered to the agent after the principal announced the result of $I T_{P}$, and a concealment contract where the agent exerts effort before $I T_{P}$ is checked. We find that concealment is superior whenever the quality of $I T_{P}$ is sufficiently low. Then, $I T_{P}$ is almost worthless under a disclosure contract, while it can still be exploited to reduce the agent's information rent under concealment. If the quality of $I T_{P}$ improves, disclosure can be superior as it allows to adjust the agent's effort to the up-dated expected quality of the project. However, even for a highly informative $I T_{P}$, concealment can be superior as it mitigates the adverse selection problem. Finally, we prove that the principal always benefits from checking $I T_{P}$ if he chooses the optimal timing of information release. In particular, he may benefit only if he does not check $I T_{P}$ until the agent reported his findings.

Keywords: information revelation, endogenous timing, hidden action, limited liability.

JEL classification: D02, D82, D86.
\end{abstract}

${ }^{*}$ We are grateful to Dominique Demougin, Christian Ewerhart, Uli Hege, Jean-Jacques Herings, Ben Jacobsen, Bettina Klaus, Ernst Maug, Gerd Muehlheusser, Martin Nell, and David Sappington. The third author gratefully acknowledges funding from the Dutch Science Foundation (NWO).

${ }^{\dagger}$ Department of Economics, Aachen University of Technology (RWTH), Templergraben 64, 52062 Aachen, Germany. Phone: 0049-241-8096155. email: feess@rwth-aachen.de.

¥Department of Economics and METEOR, University of Maastricht, P.O. Box 616, NL-6200 MD Maastricht, The Netherlands. Phone: 0031-43-3883807. email: m.walzl@algec.unimaas.nl. 


\section{Introduction}

Motivation With the amount of accessible information increasing constantly, the optimal organization of information aggregation made it to the top of the agenda. While economic theory focused on the analysis of economic relationships with a fixed distribution of information for a long time (usually referred to as the principal-agent literature), more recent research addressed the relation between information structure and optimal mechanism design. Thereby, two research questions seem to be prevalent. How does a change in the information structure influence optimal mechanism design? And does a mechanism designer always benefit from the availability of additional information? A first answer to this question is offered by the linkage principal as introduced in Milgrom and Weber (1982) according to which a principal (or auctioneer) is always better off if he links the contract (or price) to exogenous variables that are affiliated with the agent's (or bidder's) private information. In particular they investigate how affiliated instead of private values (i.e. information of bidders about each other's willingness-to-pay) changes revenue maximizing auction design. They show that open auction formats like the English auction yield higher revenues than closed formats like a second-price sealed bid auction as bidders learn about their own valuations through the published bids of their competitors. Thus the auctioneer can benefit from additional information available to the bidders if he adjusts mechanism design appropriately. ${ }^{1}$

An obviously related question is whether an auctioneer (or principal) can benefit in a similar way from additional information available to himself. This has been discussed extensively in the literature on informed principals (pioneered by Maskin and Tirole (1990) - see also the literature review at the end of the introduction). A key assumption of this literature is that the principal's information is private and non-verifiable. A recent paper by Ottaviani and Prat (2001) relates the value of information in such a setting to the respective value if the principal has access to (verifiable) information that becomes public as soon as he acquires it. In a model of monopolistic price discrimination they show that the principal (the seller) always benefits from additional information (e.g. an outsider's certification of quality) available to him - be it private or not - as long as it is correlated with the agent's (buyer's) private valuations. Moreover, they show for the private information case that it is an optimal strategy for the principal to commit himself to reveal his information before the agent chooses a contract.

This paper analyzes how these results carry over from the pure adverse selection setting to situations that (in addition) involve moral hazard. We therefore focus on principalagent relationships where an agent exerts unobservable effort to determine the quality of a project (moral hazard) and reports his findings opportunistically (adverse selection). In addition, the principal has access to information that is correlated to the agent's findings (i.e. correlated to the quality of the project). Such a setting is relevant under many practical circumstances. Initially, our analysis has been motivated by a project concerning

\footnotetext{
${ }^{1}$ This result is strengthened in Cremer and McLean (1985). They provide sufficient conditions for the information structure (i.e. the affiliation of bidders' valuations) that guarantee the existence of a mechanism that allows the auctioneer to extract all the surplus from trade.
} 
scoring systems for small enterprize loans. Loan officers who are supposed to improve and to reveal their own knowledge from relationship lending to their superiors often seem to duplicate the scoring system's result without working seriously. Hence, one may question if committing to run the scoring system (which provides verifiable information) after the loan officer's assessment is better. In a similar way, a doctor's patient, a lawyer's client or a journal editor might consider not to reveal existing expertise or reports to their contractual partner (doctor, lawyer or referee). Furthermore, consider an investor or a CEO who decides when to undertake an in-house database research complementary to the (out-sourced) work of a consulting agency. As a final example, our set-up applies to environmental auditing where an agency spends effort to investigate the security standards of a firm (i.e. detects negligent firms). Meanwhile, the government can conduct pollution measurements either to guide the agency towards suspected firms (disclosure) or to monitor the quality of the agency's findings (concealment).

Model and Main Results We analyze a model in which a principal owns an invetsment project that can be either good or bad. The principal has two possibilities to improve his knowledge about the project's quality. On the one hand, he can use a costless information technology $\left(I T_{P}\right)$ that is not subject to any kind of incentive problem. With some probability the technology correctly identifies bad projects, the respective signal is verifiable and publicly announced as soon as the principal checks it. On the other hand, the principal can hire an agent (technology $I T_{A}$ ) who may also detect bad projects with some probability depending on his unobservable effort. After having investigated the project, the agent reports his findings opportunistically to the principal. Hence, engaging the agent is not only subject to moral hazard, but also to a truth-telling (adverse selection) problem. To rule out a trivial franchise solution, the agent is assumed to have zero wealth, such that all payments from the principal to the agent are non-negative. $I T_{A}$, hence, resembles a principal-agent problem with moral hazard, adverse selection and limited liability.

Our paper addresses two main questions. First, we analyze whether access to additional (costless) information can actually harm the principal. Second, we discuss the optimal timing of information release, i.e. whether the principal should commit himself to check (and reveal) $I T_{P}$ before or after the agent exerts effort. The first option will be called a disclosure contract $\left(C^{D}\right)$ and the second one a concealment contract $\left(C^{C}\right)$. The advantage of disclosure is that the principal will hire the agent only if $I T_{P}$ has not detected a bad project as such a project will certainly fail. Furthermore, if the agent is hired, the principal can offer a contract that adjusts the agent's effort to the up-dated probability of a good project. Both is not possible under concealment. The advantage of concealment, however, is that the principal can use the signal provided by $I T_{P}$ (which is correlated with the agent's true findings) as a device to reduce the agent's rent coming from the adverse selection problem by paying positive wages if and only if the agent's report and the signal's outcome coincide.

Regardless of the contract type, the presence of moral hazard and adverse selection forces the principal to pay the agent a positive wage if he reports a good project as well as if 
he reports a bad project. Paying positive wages if the agent claims "bad" is necessary to induce effort (moral hazard problem) and paying the agent if he states "good" is required to prevent that he reports "bad" without working at all (truth-telling or adverse selection problem). Hence, the agent will receive information rents and the optimal contract is determined by a trade-off between rent-reduction and effort selection.

We derive the following set of results: First, under a disclosure contract, the principal's expected profit is not necessarily increasing in the probability that the project is good. This counterintuitive result is driven by the fact that a high percentage of good projects makes it (for any given effort level) unlikely that the agent detects bad projects. This reduces the agent's expected returns to effort (and enhances the moral hazard problem). Moreover, it increases his incentive to claim that the project is bad without working at all (e.g. it aggravates the truth-telling problem). We show that these effects may well dominate such that the principal's profit is decreasing in the probability of good projects. As a consequence, an ignorance contract where the principal does not check the (costless) $I T_{P}$ may dominate the optimal disclosure contract. The reason is that the probability for good projects is increasing if $I T_{P}$ does not detect a bad project, and this may reduce the principal's profits because of the aggravated moral hazard and adverse selection problem. Hence, remaining uninformed can be superior.

As for a comparison between disclosure and concealment, we identify several factors determining which of the contracts should be chosen. A very clear-cut result is that concealment dominates whenever the quality of $I T_{P}$ (defined by the probability $\pi_{P}$ that bad projects are identified) is sufficiently small. In these cases, $I T_{P}$ is almost worthless under a disclosure contract, while it can still be exploited to reduce the agent's information rent under concealment. On the other hand, it cannot be taken for granted that disclosure dominates whenever the quality of $I T_{P}$ is sufficiently high - at first sight this seems to be a straightforward conjecture as the cost-savings of disclosure (due to not hiring the agent) increase in $\pi_{P}$. However, as the quality of $I T_{P}$ increases, the truth-telling problem also becomes more severe (the reason again being that the agent is then more tempted to claim that a project is bad without working at all). In fact, we show that disclosure is likely to dominate only if the quality of $I T_{P}$ is in an intermediate range - if $\pi_{P}$ is too low, the advantage of not hiring the agent becomes too small, and if $\pi_{P}$ is too high, the truth-telling problem is too severe. This shows that $I T_{P}$ can also be too informative for disclosure contracts to be optimal. Finally, while an optimal disclosure contract can be dominated by a contract that ignores $I T_{P}$, we show that concealment is always better than ignorance. Therefore, additional information does not harm the principal if he chooses the optimal mechanism - here, an optimal timing of information release.

Relation to the Literature Demski and Sappington (DS 1987b) is the only paper that addresses the question of the optimal timing of information release in a principal-agent relationship. DS 1987b is an extension of Demski and Sappington (1987a) that pioneers the literature on delegated expertise. In these models, the principal delegates both the information acquisition and a subsequent implementation decision (an investment, for instance) 
to the agent because communication is prohibitively costly. In DS 1987b, the agent's unobservable effort in acquiring information is binary, and the optimal (non-binary) implementation decision depends on both the agent's and the principal's information. DS 1987b provide examples that illustrate the advantages of different timings of information release. The continuous modelling of the agent's effort costs in our paper allows for an analysis of trade-offs between the costs of implementing truth-telling and selecting an effort level and a characterization of information technologies that lead to different optimal timings of information release.

In a recent paper, Gromb and Martimort (GM 2004) discuss the optimal organization of delegated expertise. While our paper is close to their contribution with respect to the modelling of information and the basic features of the principal-agent relationship (riskneutrality, limited liability, and unobservable effort/findings), the two papers can be viewed as complements for several reasons. First of all, we do not assume an environment of delegated expertise. The principal bases his investment decision on the available information (be it his prior, $I T_{P}$ or $I T_{A}$, or both), but decides himself. Nevertheless, delegation (e.g. investment if and only if the agent does not detect a bad project) can arise endogenously in our framework. Second - and more importantly, GM 2004's focus is on the economies of scale of delegated expertise. They show that additional signals for the same agent induce diseconomies of scale (i.e. in the two signal case, agency costs are more than twice as large as in the single signal case). In contrast, an additional agent - who has access to a similar information technology - induces increasing returns to scale (i.e. agency costs for two agents are less than twice as large as in the single agent case). Hence, GM 2004 emphasize that principals benefit more from multiple experts with one signal each than from a single expert with multiple signals. Our paper, however, takes the "production-technology" of information as given and investigates their optimal exploitation. In particular, we highlight the importance of an appropriate timing of information release which is not discussed in GM2004 and thereby assess the robustness of the scale-economies they identify.

Finally, our analysis is related to the literature on informed principals developed by Maskin and Tirole (MT 1990), Maskin and Tirole (MT 1992) and Beaudry (1994) where the principal may signal his private information through a menu of contracts. The main result of MT 1990 is that the principal strictly benefits from private information with respect to his utility function. MT 1992 show that this result does not carry over to situations where the principal's private information directly enters the agent's pay-off. Chade and Silvers (2001) factor moral hazard with respect to production (and not information acquisition) into these models. They show that there are beliefs supporting a perfect Bayesian equilibrium where the principal is better off if his technology becomes less informative. The key difference between the models on informed principals and our paper is that the principal's information is private and non-verifiable in this literature whereas in our model the contract can be made directly contingent on the principal's verifiable information. Acquisition, however, makes this information publicly accessible.

Our paper is organized as follows. In section 2, we introduce the model. Section 3 derives optimal contracts for some benchmark cases such as the first best or a pure moral hazard 
setting without adverse selection. Section 4 continues with optimal contract design for a given timing of information release, while section 5 analyzes optimal timing options as a function of the informativeness of the principal's signal. Section 6 concludes with some remarks concerning the applicability and robustness of our findings.

\section{The model}

A risk-neutral principal owns a project requiring initial investment outlays of $I$. The project is of quality $q \in\{g, b\}$, and it is assumed to be "good" ( $q=g$ ) with probability $p_{0} \in(0,1)$ and "bad" $(q=b)$ with probability $\left(1-p_{0}\right)$. If it is good, it yields return $R$. If it is bad, it yields 0 . The project's expected net return is positive, i.e. $p_{0} R-I>0$. The principal can base his decision on two information technologies, $I T_{P}$ and $I T_{A}$.

The Principal's Technology $\left(I T_{P}\right)$ : The principal has costless access to an information technology $\left(I T_{P}\right)$ that yields a signal $s_{P} \in\{g, b\}$ and thereby detects bad projects with probability $\pi_{P} \in(0,1)$ (i.e. $\pi_{P}=\operatorname{Pr}\left(s_{P}=b \mid q=b\right)$ and $\operatorname{Pr}\left(s_{P}=b \mid q=g\right)=0$ ). Hence, if the signal is $s_{P}=b$ the project is certainly bad. If the signal is $s_{P}=g$ the probability of success is updated to $p_{P}>p_{0}$ where Bayesian consistency requires that $p_{P}=\frac{\operatorname{Pr}\left(\left(s_{P}=g\right) \cap(q=g)\right)}{\operatorname{Pr}\left(s_{P}=g\right)}=\frac{p_{0}}{1-\left(1-p_{0}\right) \pi_{P}}$. We will refer to $\pi_{P}$ as the quality (or informativeness) of $I T_{P}$. Note that there is no incentive problem associated with $I T_{P}$. We assume that the signal is verifiable and that it becomes common knowledge in the very moment when the principal checks the IT. ${ }^{2}$

The Agent's Technology $\left(I T_{A}\right)$ : Second, the principal can hire a risk-neutral agent who exerts unobservable effort to detect bad projects. Let $e\left(\pi_{A}\right)$ be the effort costs required to identify a bad project with probability $\pi_{A}$. Analogously to $I T_{P}$, the agent's technology sends $s_{A}=b$ in case of detection and $s_{A}=b$, otherwise. Hence, $\pi_{A}=\operatorname{Pr}\left(s_{A}=b \mid q=b\right)$ while $\operatorname{Pr}\left(s_{A}=b \mid q=g\right)=0$. We assume that $e\left(\pi_{A}\right) \in C^{3}$, that $e(0)=e^{\prime}(0)=e^{\prime \prime}(0)=0$, $e^{\prime \prime \prime}\left(\pi_{A}\right)>0 \forall \pi \in[0,1)$, and $\lim _{\pi \rightarrow 1} e^{\prime \prime \prime}\left(\pi_{A}\right)=\infty .{ }^{3} \pi_{A}$ can therefore be interpreted as the quality of $I T_{A}$. After having detected a bad project or not, the agent sends a verifiable message $m \in\{g, b\}$ to the principal. The agent's findings are private information, and the message is (thus) needs not to be truthful. The agent's reservation utility is normalized to 0 , and he is protected by limited liability in the sense that his wage must be non-negative in each state of the world.

\footnotetext{
${ }^{2}$ If the principal can check and hide the signal, this leads to a signaling game where the principal may signal his information through the contract he offers. But the principal's signal $\left(s_{P}\right)$ is binary in our model, one can easily prove that there is no separating or pooling equilibrium leading to higher profits for the principal then the profit maximizing contracts investigated below. Proof available on request.

${ }^{3}$ These assumptions ensure convexity of the principal's optimization problem, and in particular uniqueness of the optimal effort level or signal quality.
} 
Contracts The game starts at date $t=0$ with a contract offer $C$ by the principal. A contract $C$ is a collection $(T, a, W)$. $T$ (the timing) is a mapping from the set of verifiable events $E$ to the set of orderings over $E$. Once the contract has been signed, $T$ also depicts the timing of the game. a (the investment decision) is a binary choice (invest or not) defined on the set of available verifiable signals at the time of contract enforcement. $W$ (the wages) is a mapping from the set of available verifiable signals to $R_{0}^{+} .{ }^{4}$ We will refer to the principal's subgame-perfect equilibrium offers (for a given timing $T$ ) as optimal contracts. ${ }^{5}$

Timing In our setting, the (maximum) set of verifiable events consists of signing a contract (Sign), enforcing the contract or arranging payments $(E n f)$, checking the principal's signal $\left(I T_{P}\right)$, checking the agent's signal $\left(I T_{A}\right)$, and the investment decision $(\operatorname{Inv}){ }^{6}$ If the principal does not hire the agent, the obviously optimal timing is the sequence $T\left(I T_{P}, I n v\right)=\left\{I T_{P}, I n v\right\}$ as the signal is informative and costless. This defines the Null contract (denoted by $C^{0}$ ). If the principal hires the agent, (Sign) has to be before $\left(I T_{A}\right)$, and $\left(I T_{A}\right)$ before $(E n f)$. Obviously, the principal's investment decision will always take place after all information has been acquired and before the agent is payed.

Hence, we are left with four timing options: $T_{1}=\left\{\operatorname{Sign}, I T_{P}, I T_{A}, \operatorname{Inv}, \operatorname{Enf}\right\}, T_{2}=$

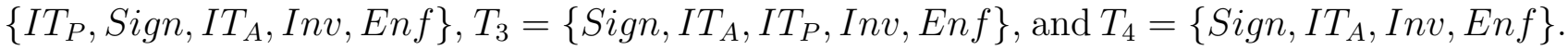

$T_{1}$ and $T_{2}$ are equivalent as the agent is risk-neutral and the principal will offer the same contract (contingent on his own signal) in both cases, so that we are left with $T_{2}, T_{3}$, and $T_{4}$. We will refer to a contract with timing $T_{2}$ as a Disclosure Contract $C^{D}$ (the principal discloses $I T_{P}$ before the agent exerts effort). A contract with timing $T_{3}$ is called a Concealment Contract $\left(C^{C}\right)$ as $I T_{P}$ is concealed until the agent reports his findings. Finally, $T_{4}$ defines an Ignorance Contract $\left(C^{I}\right)$ as $I T_{P}$ is ignored.

Investment Decision The principal's investment decision $(a)$ is a mapping from the set of (available) verifiable information to the set $\{i, n\}$ where $i$ denotes the decision to invest and $n$ not to invest. For instance, if the principal chooses to use $I T_{P}$ and $I T_{A}$, his investment decision is a mapping $a:\{g, b\} \times\{g, b\} \rightarrow\{i, n\}$.

Wages Finally, a contract specifies (weakly positive) transfers to the agent contingent on the feasible verifiable information, i.e. the signals by the information technologies and the result of the project in case of investment. For instance, if the principal checks

\footnotetext{
${ }^{4}$ Implicitly, we exclude mixed strategies for the principal (e.g. that he only checks $I T_{P}$ with a positive probability after the agent reported his findings or that he chooses different timing option with positive probability). As long as $I T_{P}$ is costless and we investigate a one-shot game, it is easy to see that such a mixed strategy can never be optimal. We return to this issue in the concluding section.

${ }^{5}$ Our assumptions on $e\left(\pi_{A}\right)$ ensure that subgame-perfection selects a unique contract offer (with the only exception being section 3.3.3).

${ }^{6}$ Hence, we implicitly assume that the principal can not check his signal between the agent's effort and reporting decision. As effort is unobservable, this assumption seems justified. Moreover, it can be shown that the principal will never be better off if he considers this timing of events. Proof available on request.
} 
$I T_{P}$ and $I T_{A}$ and the signal configuration suggests investment, wages are a mapping $W$ : $\{g, b\} \times\{g, b\} \times\{g, b\} \rightarrow R_{0}^{+}$denoted by $W\left(s_{P}, s_{A}, q\right)$ where the first argument depicts the result of $I T_{P}$, the second $I T_{A}$, and the last whether the project was successful or not. If the principal decides to ignore some sources of information, feasible wage schemes are defined accordingly. We denote the agent's expected utility by $U$ and the principal's expected profits by $\Phi$.

\section{Benchmarks}

We start the analysis and comparison of the principal's options by investigating three benchmark contracts, namely the Null Contract $C^{0}$ where the principal does not hire the agent, the First Best Contract $C^{F}$ where the principal himself has access to $I T_{A}$, and the case without adverse selection (or truth-telling) problem where the agent's findings are verifiable.

\subsection{Null Contract}

If the principal decides not to hire the agent, he will obviously check $I T_{P}$ before he decides upon investment. Optimal actions, transfers, and profits are depicted in the following Lemma. ${ }^{7}$

Lemma 1. (i) $\pi_{A}^{0}=0$, (ii) $a^{0}\left(s_{P}\right)=i$ if and only if $s_{P}=g$. (iii) $\Phi^{0}=\operatorname{Pr}\left(s_{P}=\right.$ $g)\left(p_{P} R-I\right)=p_{0} R-\operatorname{Pr}\left(s_{p}=g\right) I$. (iv) $\frac{d \Phi^{0}}{d \pi_{P}}>0$.

All proofs are in the Appendix.

Of course, the principal invests if and only if $s_{P}=g$, and his profit increases in the quality of $I T_{P}$ (i.e. in $\pi_{P}$ ).

\section{2 $\quad$ First Best}

In the first best $\left(C^{F}\right)$ the principal checks $I T_{P}$ and uses $I T_{A}$ if and only if $s_{P}=g$. In this case, he maximizes $p_{P}(R-I)-\left(1-p_{P}\right)\left(1-\pi_{A}\right) I-e\left(\pi_{A}\right)$ w.r.t. $\pi_{A}$ which yields Lemma $2 .^{8}$

Lemma 2. (i) $\pi_{A}^{F}$ solves $\left(1-p_{P}\right) I=e^{\prime}\left(\pi_{A}^{F}\right)$. (ii) $a^{F}\left(s_{P}, s_{A}\right)=i$ if and only if $s_{P}=s_{A}=g$. (iii) $\Phi^{F}=\operatorname{Pr}\left(s_{P}=g\right)\left(p_{P} R-I+\left(1-p_{P}\right) \pi_{A}^{F} I-e\left(\pi_{A}^{F}\right)\right)$. (iv) $\frac{d \Phi^{F}}{d \pi_{P}}>0$.

Note that $I T_{A}$ is only checked if $s_{P}=g$. Moreover, the assumptions on $e\left(\pi_{A}\right)$ guarantee that $\pi_{A}^{F}>0$ in this case. Hence, the first best contract is of disclosure type.

\footnotetext{
${ }^{7}$ We denote optimal transfers, actions and equilibrium profits for $C^{0}$ by superscript 0 .

${ }^{8}$ We denote optimal transfers, actions and equilibrium profits for $C^{F}$ by superscript $F$.
} 


\subsection{Pure Moral Hazard}

To distinguish the impact of moral hazard (hidden action) and truth-telling (hidden information) in our setting, we assume throughout this section that the agent's findings are observable and verifiable (i.e. $m=s_{A}$ ) such that only only a moral hazard problem remains. ${ }^{9}$

\subsubsection{Ignorance}

If the principal does not check $I T_{P}$ and hires the agent, observability of $s_{A}$ implies that he invests if and only if $s_{A}=g$. Hence, an ignorance contract specifies $W(g, q)$ for $q \in\{g, b\}$ and $W(b)$. For such a contract, the agent's utility for a given signal quality $\pi_{A}$ (denoted by $\left.U\left(\pi_{A}\right)\right)$ is given by

$$
U\left(\pi_{A}\right)=p_{0} W(g, g)+\left(1-p_{0}\right)\left(\pi_{A} W(b)+\left(1-\pi_{A}\right) W(g, b)\right)-e\left(\pi_{A}\right)
$$

which yields the agent's incentive compatibility constraint (ICC)

$$
\left(1-p_{0}\right)(W(b)-W(g, b))=e^{\prime}\left(\pi_{A}\right) .
$$

The principal's optimization program is ${ }^{10}$

$$
\begin{array}{ll}
\max & \left(p_{0}(R-I-W(g, g))-\left(1-p_{0}\right)\left(\pi_{A} W(b)+\left(1-\pi_{A}\right)(I+W(g, b))\right)\right. \\
& \text { s.t.Eqn. }(2), W(g, g) \geq 0, W(g, b) \geq 0, W(b) \geq 0 .
\end{array}
$$

The solution to this program can be characterized as follows. ${ }^{11}$

Lemma 3. (i) $\widetilde{\pi}_{A}^{I}$ solves $\left(1-p_{0}\right) I=e^{\prime}\left(\widetilde{\pi}_{A}^{I}\right)+\widetilde{\pi}_{A}^{I} e^{\prime \prime}\left(\widetilde{\pi}_{A}^{I}\right)$. (ii) $\widetilde{W}^{I}(g, g)=\widetilde{W}^{I}(g, b)=0$ and $\widetilde{W}^{I}(b)=\frac{e^{\prime}\left(\widetilde{\pi}_{A}^{I}\right)}{1-p_{0}}$. (iii) $\widetilde{a}^{I}\left(s_{A}\right)=i$ if and only if $s_{A}=g$. (iv) $\widetilde{\Phi}^{I}=p_{0} R-I+\left(1-p_{0}\right) \widetilde{\pi}_{A}^{I} I-$ $\tilde{\pi}_{A}^{I} e^{\prime}\left(\tilde{\pi}_{A}^{I}\right)$.

First note that the agent will always be hired as his marginal disutility from effort is zero at $\pi_{A}=0$. As the agent's incentive compatibility constraint (Eqn. (2)) is the only non-trivial constraint, and as payments contingent on successful projects do not influence the agent's effort, the optimal contract offers only compensation for the detection of bad projects. The principal's profit as depicted in (iv) consists of the returns to investment $\left(p_{0} R-I\right)$, the benefits (or cost savings) from hiring the agent $\left(\left(1-p_{0}\right) \widetilde{\pi}_{A}^{I}\right)$, and the agency costs $\left(\tilde{\pi}_{A}^{I} e^{\prime}\left(\tilde{\pi}_{A}^{I}\right)\right)$.

\footnotetext{
${ }^{9}$ Optimal contracts, actions, transfers, and profits for the pure moral hazard case are denoted by () .

${ }^{10}$ Note that $\frac{d^{2} U\left(\pi_{A}\right)}{d \pi_{A}^{2}}<0$ and that $U\left(\pi_{A}\right) \geq 0$ for $\pi_{A}=0$ such that the agent's (ICC) ensures $U>0$. Thus, the agent's participation constraint $(U>0)$ is ensured by (ICC) and can be neglected in all optimization programs.

${ }^{11}$ We denote optimal contracts and actions for ignorance contract by superscript $I$.
} 


\subsubsection{Disclosure}

If the principal checks $I T_{P}$ before the agent signs a contract, he will never hire him if $s_{P}=b$. Hence, we can restrict attention to $s_{P}=g$. Then, the agent's expected utility and the incentive compatibility constraint can be elicited from Eqn. (1) and Eqn. (2) by substituting $p_{P}$ (the updated success-probability) for $p_{0}$, and by specifying transfers as follows. The principal does not pay anything if $s_{P}=b$, pays $W(g, g, g)$ if $s_{P}=s_{A}=q=g$, $W(g, b)$ if $s_{P}=g$, and $s_{A}=b$ (recall that $s_{A}$ is observable such that there is no point in investing in this case), and $W(g, g, b)$ if $s_{P}=s_{A}=g$ and $q=b$. The principal's optimization program is

$$
\begin{array}{ll}
\max & \operatorname{Pr}\left(s_{P}=g\right)\left(p_{P}(R-I-W(g, g, g))\right. \\
& \left.-\left(1-p_{P}\right)\left(\pi_{A} W(g, b)+\left(1-\pi_{A}\right)(I+W(g, g, b))\right)\right) \\
& \text { s.t.Eqn. }(2)^{\prime}, W(g, g, g) \geq 0, W(g, b) \geq 0, W(b) \geq 0 .
\end{array}
$$

where Eqn.(2)' denotes the incentive compatibility constraint as in Eqn.(2) with the updated success probability $p_{P}$ instead of $p_{0}$. The respective solution can be characterized as follows. ${ }^{12}$

Lemma 4. $\widetilde{\pi}_{A}^{D}$ solves $\left(1-p_{P}\right) I=e^{\prime}\left(\widetilde{\pi}_{A}^{D}\right)+\widetilde{\pi}_{A}^{D} e^{\prime \prime}\left(\widetilde{\pi}_{A}^{D}\right)$. (ii) $\widetilde{W}^{D}(g, g, g)=\widetilde{W}^{D}(g, g, b)=0$ and $\widetilde{W}^{D}(g, b)=\frac{e^{\prime}\left(\widetilde{\pi}_{A}^{D}\right)}{1-p_{P}}$. (iii) $\widetilde{a}^{D}\left(s_{P}, s_{A}\right)=i$ if and only if $s_{P}=s_{A}=g$. (iv) $\widetilde{\Phi}^{D}=\operatorname{Pr}\left(s_{P}=\right.$ $g)\left(p_{P} R-I+\left(1-p_{P}\right) \widetilde{\pi}_{A}^{D} I-\widetilde{\pi}_{A}^{D} e^{\prime}\left(\widetilde{\pi}_{A}^{D}\right)\right)$. (v) $\frac{d \widetilde{\Phi}^{D}}{d \pi_{P}}>0$.

A comparison of Lemma 2 (i) and 4 (i) shows that the signal quality (or effort) chosen in the pure moral hazard case is smaller than in the first best solution (due to the binding limited liability constraint). Moreover, equilibrium efforts in the disclosure contract are lower than in the ignorance contract. ${ }^{13}$ Finally, the principal is strictly better off by checking $I T_{P}$ in advance than by ignoring it as $\widetilde{\Phi}^{D}$ is monotone increasing in $\pi_{P}$, and as an ignorance contract resembles $\pi_{P}=0$.

\subsubsection{Concealment}

If the principal checks $I T_{P}$ after the agent's investigation results are observed, he invests if and only if $s_{P}=s_{A}=g$. Hence, a contract specifies $W(g, g, g)$ (agent and principal do not detect a bad project and the project turns out to be successful), $W(g, g, b)$ (agent and principal do not detect a bad project and the project fails), and the wages if at least one technology detects a bad project $(W(g, b), W(b, g)$, and $W(b, b))$. For such a contract, the agent's expected utility for a given signal quality $\pi_{A}$ is

$$
\begin{aligned}
U\left(\pi_{A}\right)= & p_{0} W(g, g, g)+\left(1-p_{0}\right)\left(\pi_{A} \pi_{P} W(b, b)+\pi_{P}\left(1-\pi_{A}\right) W(b, g)\right. \\
& \left.+\left(1-\pi_{P}\right) \pi_{A} W(g, b)+\left(1-\pi_{P}\right)\left(1-\pi_{A}\right) W(g, g, b)\right)-e\left(\pi_{A}\right)
\end{aligned}
$$

\footnotetext{
${ }^{12}$ We denote optimal contracts and actions under policy $\mathrm{D}$ with superscript $D$.

${ }^{13}$ To see this note that $\left(1-p_{P}\right)<\left(1-p_{0}\right)$.
} 
which yields the agent's incentive compatibility constraint

$$
\left(1-p_{0}\right)\left(\pi_{P}(W(b, b)-W(b, g))+\left(1-\pi_{P}\right)(W(g, b)-W(g, g, b))=e^{\prime}\left(\pi_{A}\right) .\right.
$$

The principal's optimization program is

$$
\begin{aligned}
& \max \quad p_{0}(R-I-W(g, g, g))-\left(1-p_{0}\right)\left(\pi_{A}\left(1-\pi_{P}\right) W(g, b)\right. \\
& \left.+\left(1-\pi_{A}\right) \pi_{P} W(b, g)+\pi_{A} \pi_{P} W(b, b)+\left(1-\pi_{0}\right)\left(1-\pi_{A}\right)(I+W(g, g, b))\right) \\
& \text { s.t.Eqn.(4), } W(g, g, g) \geq 0, W(g, g, b) \geq 0, W(g, b) \geq 0, W(b, g) \geq 0 \text {. }
\end{aligned}
$$

Optimal contracts can be characterized as follows. ${ }^{14}$

Lemma 5. (i) $\widetilde{\pi}_{A}^{C}$ solves $\left(1-p_{0}\right)\left(1-\pi_{P}\right) I=\widetilde{\pi}_{A}^{C} e^{\prime \prime}\left(\widetilde{\pi}_{A}^{C}\right)+e^{\prime}\left(\widetilde{\pi}_{A}^{C}\right)$. (ii) $\widetilde{W}^{C}(g, g, g)=$ $\widetilde{W}^{C}(g, g, b)=\widetilde{W}^{C}(b, g)=0$ and $\left(1-p_{0}\right)\left(\pi_{P} \widetilde{W}^{C}(b, b)+\left(1-\pi_{P}\right) \widetilde{W}^{C}(g, b)\right)=e^{\prime}\left(\widetilde{\pi}_{A}^{C}\right)$. (iii) $\widetilde{a}^{C}\left(s_{P}, s_{A}\right)=i$ if and only if $s_{P}=s_{A}=g$. (iv) $\widetilde{\Phi}^{C}=p_{0} R-I+\left(1-p_{0}\right) \pi_{P} I+\left(1-p_{0}\right)(1-$ $\left.\pi_{P}\right) \widetilde{\pi}_{A}^{C} I-\widetilde{\pi}_{A}^{C} e^{\prime}\left(\widetilde{\pi}_{A}^{C}\right)$. (v) $\frac{d \widetilde{\Phi}^{C}}{d \pi_{P}}>0$.

As in the optimal ignorance and disclosure contracts, the agent is only compensated for the detection of bad projects. Note that any combination of the respective wages $W(g, b)$ and $W(b, b)$ fulfilling Eqn. (4) can be part of the optimal contract. The optimal signal quality $\widetilde{\pi}^{C}$ is smaller than $\widetilde{\pi}^{D} \cdot{ }^{15}$

\subsubsection{Endogenous Timing}

For the pure moral hazard case, an inspection of the respective efforts and profits yields the following comparison of the three different contract types.

Proposition 1. (i) $\pi_{A}^{F}>\widetilde{\pi}_{A}^{I}>\widetilde{\pi}_{A}^{D}>\widetilde{\pi}_{A}^{C}>0$. (ii) $\Phi^{F}>\widetilde{\Phi}^{D}>\widetilde{\Phi}^{C}>\widetilde{\Phi}^{I}>0$.

Proposition 1 shows the following trade-off between the marginal benefits of effort implementation and an efficient use of $I T_{P}$ : First, recall from Lemma 3(i), 4(i), and 5(i)) that marginal costs of effort implementation are identical for the three contract types they consist of the marginal effort costs themselves $\left(e^{\prime}\left(\pi_{A}\right)\right)$ and the additional marginal costs due to moral hazard under limited liability - the marginal agency costs $\left(\pi_{A} e^{\prime \prime}\left(\pi_{A}\right)\right)$. The principal's marginal benefits from the agent's effort, however, differ for the three contract types. In a disclosure contract, marginal benefits are smaller than for ignorance as the agent is only hired if $s_{P}=g$ (and the probability to detect a bad project shrinks to $\left.\left(1-p_{P}\right)<\left(1-p_{0}\right)\right)$. In a concealment contract, marginal benefits are further reduced as the agent provides valuable information only if he detects a bad project which has not been detected by $I T_{P}$ (which happens with probability $\left(1-p_{0}\right)\left(1-\pi_{P}\right)<\left(1-p_{P}\right)$ ). This

\footnotetext{
${ }^{14}$ We denote optimal contracts and actions for a concealment contract by superscript $C$.

${ }^{15}$ To see this note that $\left(1-p_{P}\right)>\operatorname{Pr}\left(s_{P}=g\right)\left(1-p_{P}\right)=\left(1-p_{0}\right)\left(1-\pi_{P}\right)$.
} 
effect drives Part (i) and if it it were for marginal benefits alone, ignorance would dominate disclosure, and disclosure would dominate concealment with respect to the principal's profits.

But on the other hand, the three contract types make different use of $I T_{P}$ both with respect to the decision to hire the agent and with respect to investment. Under disclosure and concealment contracts, the principal invests for the same signal configurations such that these policies only differ with respect to the hiring decision. As the agent is hired too often under concealment (in particular if $s_{P}=b$ ), disclosure is less costly than concealment. Under ignorance, however, the principal invests more into unsuccessful projects than under disclosure and concealment, and he hires the agent as often as under concealment. Hence, with respect to the use of $I T_{P}$, disclosure is superior to concealment which dominates ignorance from the principal's point of view.

Taking both effects (marginal benefits of effort and use of $I T_{P}$ ) into account obviously implies that disclosure dominates concealment. Moreover, Proposition 1 (ii) demonstrates that the latter effect is stronger than the former when comparing ignorance and disclosure.

\section{Optimal Contracts}

We are now far enough along to consider the complete model with unobservable findings (or adverse selection), and we will show that the profit-ranking as depicted in Proposition 1 is no longer valid. The following Lemma streamlines the analysis.

Lemma 6. (i) If the principal hires the agent, he does not invest whenever the agent sends message $m=b$. (ii) If the agent chooses $\pi_{A}>0$, he tells the truth.

Lemma 6 implies that the agent's and principal's optimization program is identical to the pure moral hazard situation in the previous section except for truth-telling constraints. We will again consider the three contract types ignorance, disclosure, and concealment.

\subsection{Ignorance}

If the principal does not check $I T_{P}$ and hires the agent, Lemma 6 implies that he invests if and only if $s_{A}=g$. Hence, a contract specifies transfers $W(g, q)$ for $q \in\{g, b\}$ and $W(b)$. The agent's utility from this contract for a given signal quality $\left(U\left(\pi_{A}\right)\right)$ is as in Eqn. (1), and the incentive compatibility constraint is identical to Eqn. (2). Finally, the agent reports truthfully (after spending a given effort $e\left(\pi_{A}\right)$ ) if this weakly dominates not working at all and always reporting $s_{A}=g$, i.e.

$$
U\left(\pi_{A}\right) \geq p_{0} W(g, g)+\left(1-p_{0}\right) W(g, b)
$$

and not working at all and always reporting $s_{A}=b$, i.e.

$$
U\left(\pi_{A}\right) \geq W(b) .
$$


Note that the right-hand-side of Ineq. (5) is just the agent's expected utility $U\left(\pi_{A}\right)$ for $\pi_{A}=0$. Hence, Ineq. (5) is implied by incentive compatibility. This given, the principal's optimization program is

$$
\begin{array}{ll}
\max & p_{0}(R-I-W(g, g))-\left(1-p_{0}\right)\left(\pi_{A} W(b)+\left(1-\pi_{A}\right)(I+W(g, b))\right. \\
& \text { s.t.Eqn.(2), Ineq.(6), } W(g, g) \geq 0, W(g, b) \geq 0, W(b) \geq 0
\end{array}
$$

which yields

Lemma 7. (i) $\pi_{A}^{I}$ solves $\left(1-p_{0}\right) I=\frac{e^{\prime \prime}\left(\pi_{A}^{I}\right)}{1-p_{0}}+e^{\prime}\left(\pi_{A}^{I}\right)$. (ii) $W^{I}(g, b)=0, W^{I}(b)=\frac{e^{\prime}\left(\pi_{A}^{I}\right)}{1-p_{0}}$, and $W^{I}(g, g)=\frac{1}{p_{0}}\left(\left(1-\left(1-p_{0}\right) \pi_{A}^{I}\right) W^{I}(b)+e\left(\pi_{A}^{I}\right)\right)$. (iii) $a^{I}\left(s_{A}\right)=i$ if and only if $m=g$. (iv) $\Phi^{I}=p_{0} R-I+\left(1-p_{0}\right) \pi_{A}^{I} I-\frac{e^{\prime}\left(\pi_{A}^{I}\right)}{1-p_{0}}-e\left(\pi_{A}^{I}\right)$.

Besides paying for the detection of bad projects to mitigate the moral hazard problem, the principal now has to offer compensations for reporting good projects to satisfy the truth-telling constraint Ineq. (6). Otherwise, the agent would always report that he has detected a bad project. In an optimal contract the truth-telling constraint is binding. Note that the principal pays positive wages if message $m=g$ is send only if the project proves successful (i.e. $W(g, b)=0$ ) as this relaxes the incentive compatibility constraint. Since the truth-telling constraint increases marginal costs of effort implementation, the principal implements a lower effort, so that we have $\pi_{A}^{I}<\widetilde{\pi}_{A}^{I}$ and $\Phi_{A}^{I}<\widetilde{\Phi}_{A}^{I}$. The strict increase in agency costs compared to the pure moral hazard case can be seen in a comparison of the terms proportional to $e^{\prime}\left(\pi_{A}\right)$ in Lemma 3 (iv) and Lemma 7 (iv).

\subsection{Disclosure}

As in subsection 3.3.2, the principal does not hire the agent if $s_{P}=b$. Hence, a disclosure contract again specifies $W(g, g, g), W(g, b)$ and $W(g, g, b)$. The agent's utility and his (ICC) can again be derived from the ignorance contract by substituting $p_{P}$ for $p_{0}$, and by changing the transfers accordingly. Hence,

$$
U\left(\pi_{A}\right)=p_{P} W(g, g, g)+\left(1-p_{P}\right)\left(\pi_{A} W(g, b)+\left(1-\pi_{A}\right) W(g, g, b)\right)-e\left(\pi_{A}\right)
$$

which yields the agent's (ICC)

$$
\left(1-p_{P}\right)(W(g, b)-W(g, g, b))=e^{\prime}\left(\pi_{A}\right) .
$$

Honest reporting now requires

$$
U\left(\pi_{A}\right) \geq p_{P} W(g, g, g)+\left(1-p_{P}\right) W(g, b)
$$

and

$$
U\left(\pi_{A}\right) \geq W(g, b) .
$$


As in subsection 4.1, Ineq. (9) is implied by incentive compatibility. This given, the principal's optimization program is

$$
\begin{array}{ll}
\max & \operatorname{Pr}\left(s_{P}=g\right)\left(p_{P}(R-I-W(g, g, g))\right. \\
& \left.-\left(1-p_{P}\right)\left(\pi_{A} W(g, b)+\left(1-\pi_{A}\right)(I+W(g, g, b))\right)\right) \\
& \text { s.t.Eqn.(8), Ineq. }(10), W(g, g, g) \geq 0, W(g, g, b) \geq 0, W(g, b) \geq 0
\end{array}
$$

which leads to

Lemma 8. (i) $\pi_{A}^{D}$ solves $\left(1-p_{P}\right) I=\frac{e^{\prime \prime}\left(\pi_{A}^{D}\right)}{1-p_{P}}+e^{\prime}\left(\pi_{A}^{D}\right)$. (ii) $W^{D}(g, g, b)=0, W^{D}(g, b)=$ $\frac{e^{\prime}\left(\pi_{A}^{D}\right)}{1-p_{P}}$, and $W^{D}(g, g, g)=\frac{1}{p_{P}}\left(\left(1-\left(1-p_{P}\right) \pi_{A}^{D}\right) W^{D}(g, b)+e\left(\pi_{A}^{D}\right)\right)$. (iii) $a^{I}\left(s_{P}, s_{A}\right)=i$ if and only if $m=s_{P}=g$. (iv) $\Phi^{D}=\operatorname{Pr}\left(s_{P}=g\right)\left(p_{P} R-I+\left(1-p_{P}\right) \pi_{A}^{D} I-\frac{e^{\prime}\left(\pi_{A}^{D}\right)}{1-p_{P}}-e\left(\pi_{A}^{D}\right)\right)$. (v) $\frac{d \Phi}{d \pi_{P}}=\left(1-p_{0}\right)\left(1-\pi_{A}^{D}\right) I+e^{\prime}\left(\pi_{A}^{D}\right) \frac{\left(1-p_{0}\right)\left(1-2 p_{P}\right)}{\left(1-p_{P}\right)^{2}}+\left(1-p_{0}\right) e\left(\pi_{A}^{D}\right)$. (vi) Suppose that $p_{P} \leq 1 / 2$, then $\frac{d \Phi^{D}}{d \pi_{P}}>0$. (vii) Suppose that $p_{0}>1 / 2$, then there exists $e(\pi)$ and $\epsilon>0$ such that $\frac{d \Phi^{D}}{d \pi_{P}}<0$ if $\pi_{P}<\epsilon$.

For the same reasons as in the optimal ignorance contract, the principal offers compensations only if the agent reports a bad project or if he reports a good project which turns out to be successful (see (ii)). As in the pure moral hazard case, marginal benefits of effort are lower in the disclosure case than for ignorance as the probability of bad projects shrinks from $\left(1-p_{0}\right)$ to $\left(1-p_{P}\right)$ (see the left-hand-side of Lemma 7(i) and Lemma 8(i)). However, marginal costs of effort implementation are no longer identical (as they were in the pure moral hazard case). An inspection of Lemma 7(iv) and Lemma 8(iv)) reveals that (for any given signal quality $\pi_{A}$ ) agency costs due to limited liability and truth-telling for the optimal ignorance contract $\left(\frac{e^{\prime}\left(\pi_{A}\right)}{1-p_{0}}\right)$ are smaller than agency costs for the optimal disclosure contract $\left(\frac{e^{\prime}\left(\pi_{A}\right)}{1-p_{P}}\right)$. The smaller the probability of a bad project (i.e. the probability to get compensated with $W^{I}(b)$ or $\left.W^{D}(g, b)\right)$, the more tempting is the option to always announce $m=b$ (getting this compensation for sure). Therefore, the update from $p_{0}$ to $p_{P}$ in the disclosure contract does not only reduce marginal benefits from effort (as in the pure moral hazard case) but also tightens the truth-telling constraint and thereby increases marginal effort costs. As a consequence $\pi_{A}^{I}>\pi_{A}^{D}$. (vii) shows that this effect may well lead to an overall profit-reducing effect of an improvement of $I T_{P}$ (i.e. $\frac{d \Phi^{D}}{d \pi_{P}}<0$ ). $\frac{d \Phi^{D}}{d \pi_{P}}$ (as depicted in $\left.(\mathrm{v})\right)$ is a sum of three contributions. $\left(1-p_{0}\right)\left(1-\pi_{A}^{D}\right) I$ is the marginal cost saving of no investment, $\left(1-p_{0}\right) e\left(\pi_{A}^{D}\right)$ is the marginal cost saving of not hiring the agent, and $e^{\prime}\left(\pi_{A}^{D}\right) \frac{\left(1-p_{0}\right)\left(1-2 p_{P}\right)}{\left(1-p_{P}\right)^{2}}$ is the marginal cost of effort implementation. While the first two terms are strictly positive, the third one is negative if and only if $p_{P}>1 / 2$ (see (vi)). In the Appendix we provide an example showing that the latter effect may well dominate such that $\frac{d \Phi^{D}}{d \pi_{P}}<0$ even if $I T_{P}$ is rather uninformative. The crucial feature of the example is that the agent's effort is relatively cheap (due to a flat $e\left(\pi_{A}\right)$ for $\pi_{A} \in[0,1)$ ). Then, cost-savings due to not hiring the agent are small and costs are mainly driven by 
the implementation of truth-telling. In such a situation, Lemma 8 (vii) shows that better information (higher $\pi_{P}$ ) can be harmful for the principal.

\subsection{Concealment}

By definition of a concealment contract, the signal is checked after the agent's report. Given Lemma 6, any concealment contract specifies transfers $W(g, g, g), W(g, g, b), W(g, b)$, $W(b, g)$, and $W(b, b)$. The agent's expected utility for a given signal quality $\pi_{A}$ is given by Eqn. (3) and the agent's (ICC) is Eqn.(4). To prevent that the agent always claims that the project is good,

$$
U\left(\pi_{A}\right) \geq p_{0} W(g, g, g)+\left(1-p_{0}\right)\left(\pi_{P} W(b, g)+\left(1-\pi_{P}\right) W(g, g, b)\right)
$$

must hold which is again implied by (ICC). Not always claiming the project to be bad requires

$$
U\left(\pi_{A}\right) \geq p_{0} W(g, b)+\left(1-p_{0}\right)\left(\pi_{P} W(b, b)+\left(1-\pi_{P}\right) W(g, b)\right) .
$$

This given, the principal's optimization program is

$$
\begin{array}{ll}
\max & p_{0}(R-I-W(g, g, g))-\left(1-p_{0}\right)\left(\pi_{A}\left(1-\pi_{P}\right) W(g, b)\right. \\
& \left.+\left(1-\pi_{A}\right) \pi_{P} W(b, g)+\pi_{A} \pi_{P} W(b, b)+\left(1-\pi_{P}\right)\left(1-\pi_{A}\right)(I+W(g, g, b))\right) \\
& \text { s.t.Eqn.(4), Eqn.(12),W(g,g,g) } \geq 0, W(g, g, b) \geq 0, W(g, b) \geq 0, W(b, g) \geq 0
\end{array}
$$

which leads to

Lemma 9. (i) $\pi_{A}^{C}$ solves $\left(1-p_{0}\right)\left(1-\pi_{P}\right) I=e^{\prime \prime}\left(\pi_{A}^{C}\right)+e^{\prime}\left(\pi_{A}^{C}\right)$. (ii) $W^{C}(g, g, b)=W^{C}(b, g)=$ $W^{C}(g, b)=0, W^{C}(b, b)=\frac{e^{\prime}\left(\pi_{A}^{C}\right)}{\left(1-p_{0}\right) \pi_{P}}$, and $W^{D}(g, g, g)=\frac{1}{p_{0}}\left(\left(1-p_{0}\right)\left(1-\pi_{A}\right) \pi_{P} W^{D}(b, b)+\right.$ $\left.e\left(\pi_{A}^{C}\right)\right)$. (iii) $a^{I}\left(s_{P}, s_{A}\right)=i$ if and only if $m=s_{P}=g$. (iv) $\Phi^{C}=p_{0} R-I+\left(1-p_{0}\right) \pi_{P} I+$ $\left(1-p_{0}\right)\left(1-\pi_{P}\right) \pi_{A} I-e^{\prime}\left(\pi_{A}^{C}\right)-e\left(\pi_{A}^{C}\right)$. (v) $\frac{d \Phi}{d \pi_{P}}>0$.

In a concealment contract, the principal uses $I T_{P}$ as a stochastic signal for the agent's findings and pays him for the detection of bad projects if and only if $s_{P}=b$ - in contrast to the pure moral hazard case where both $W(b, b)$ and $W(g, b)$ could be positive in an optimal contract. Intuitively, truth-telling (Ineq. (12)) is easier to satisfy for $W(g, b)=0$ while both $W(b, b)$ and $W(g, b)$ are equally effective in securing incentive compatibility. A comparison of Lemma $5(\mathrm{i})$ and $9(\mathrm{i})$ shows that the marginal returns to effort (1 $\left.p_{0}\right)\left(1-\pi_{P}\right) I$ are the same as in the pure moral hazard case. Marginal costs of effort implementation, however, increase due to the binding truth-telling constraint (see the right-hand-side of Lemma 5(i) and 9(i))). For concealment, the increase in agency costs due to truth-telling (for a given effort level) is strictly smaller than for disclosure and ignorance as the signal provided by $I T_{P}$ can be used more effectively to reduce the agent's information rent. 


\section{Optimal Timing of Information Release}

\subsection{Inferiority of the Null Contract}

An inspection of the respective first order conditions shows that the principal is always better off by a disclosure or concealment contract with a strictly positive effort level then by not hiring the agent.

Lemma 10. (i) $\Phi^{D}>\Phi^{0}$. (ii) $\Phi^{C}>\Phi^{0}$.

This follows immediately from the assumption that $e^{\prime \prime}\left(\pi_{A}\right)=0$ for $\pi_{A}=0$ so that both marginal effort costs and marginal agency costs are zero for vanishing signal quality. We discuss the relevance of this assumption in the concluding section.

\subsection{Inferiority of Ignorance}

As $I T_{P}$ is costless for the principal, one could conjecture that it can never be optimal to ignore this signal. In fact, the optimal ignorance contract is always inferior to concealment. However, as stated in the following proposition, ignorance may be superior to disclosure.

Proposition 2. (i) $\Phi^{C}>\Phi^{I}$. (ii) Suppose that $p_{P} \leq 1 / 2$, then $\Phi^{D}>\Phi^{I}$. (iii) Suppose that $p_{0}>1 / 2$, then there exists $e(\pi)$ and $\epsilon>0$ such that $\Phi^{D}<\Phi^{I}$ if $\pi_{P}<\epsilon$.

For part (i) of the Proposition, recall that concealment already dominated ignorance in the pure moral hazard case (due to the more efficient use of $I T_{P}$ ). The additional truth-telling problem only reinforces this effect as the increase in costs of effort implementation due to the binding truth-telling constraint is smaller under concealment. The comparison to disclosure (see (ii) and (iii)), however, is more subtle due to the non-monotonicity of $\Phi^{D}$ in $\pi_{P}$ (see Lemma 8 (vi) and (vii)). As $\lim _{\pi_{P} \rightarrow 0} \Phi^{D}=\Phi^{I}$, a sufficient condition for $\Phi^{D}>\Phi^{I}$ is $\frac{d \Phi^{D}}{d \pi_{P}}>0$ which is - according to Lemma $8(\mathrm{vi})$ - guaranteed by $p_{P} \leq 1 / 2$. On the other hand, the example provided in Appendix 2 shows that $\frac{d \Phi^{D}}{d \pi_{P}}$ can be negative for $\pi_{P}$ small enough. This implies that $\Phi^{D}<\Phi^{I}$ in a neighborhood of $\pi_{P}=0$. Intuitively, the effort-cost function provided in the example is sufficiently flat as to implement a high effort level at low costs. Hence, the advantages of disclosure (cost savings from not hiring the agent and effort adjustments to new available information) are less important compared to the costs of truth-telling that increase in $\pi_{P}$ (see discussion of Lemma 8). This drives ignorance superior to disclosure.

\subsection{Superiority of Disclosure?}

In the pure moral hazard case disclosure outperformed all other contract types. The advantage of disclosure compared to concealment is that the agent is not hired if the signal is bad, and that the agent's effort can be adjusted to the updated probability that the project is good. Now, there is a countervailing effect - concealment contracts allow to 
mitigate the truth-telling problem. In the following, we provide a sufficient condition for the superiority of disclosure.

Proposition 3. Suppose $p_{0}<1 / 4$ and $\pi_{P} \in\left[\frac{1-\sqrt{1-4 p_{0}}}{2\left(1-p_{0}\right)}, \frac{1+\sqrt{1-4 p_{0}}}{2\left(1-p_{0}\right)}\right]$. Then $\Phi^{D}>\Phi^{C}>\Phi^{I}$.

The comparison of disclosure and concealment is complicated by the fact that the optimal signal qualities are different. To get clear-cut results for the superiority of disclosure, we therefore rely on cases where disclosure beats concealment even if the principal implements the suboptimal signal quality $\pi_{A}^{C}$ in a disclosure contract. The profit difference between disclosure and concealment then consists only of two terms: First, $e\left(\pi_{A}\right)\left(1-\operatorname{Pr}\left(s_{P}=g\right)\right)=$ $e\left(\pi_{A}\right)\left(1-p_{0}\right) \pi_{P}$ expresses the more efficient use of $I T_{P}$. This term is increasing in $\pi_{P}$ and captures the intuitive advantage of disclosure for informative (high $\pi_{P}$ ) technologies. The second term, $e^{\prime}\left(\pi_{A}\right) \frac{1-p_{P}-\operatorname{Pr}\left(s_{P}=g\right)}{1-p_{P}}=e^{\prime}\left(\pi_{A}\right)\left(1-\frac{\left(1-\left(1-p_{0}\right) \pi_{P}\right)^{2}}{\left(1-p_{0}\right)\left(1-\pi_{P}\right)}\right)$ is the difference in the costs of effort implementation. For $p_{0}>1 / 4$ this term is negative such that disclosure can only be superior due to adjusted effort selection. For $p_{0}<1 / 4$, however, this contribution is positive around a local maximum at some $\pi_{P} \in(0,1)$ (i.e. in the interval in the Proposition). Intuitively, if $\pi_{P}$ is low, a disclosure contract can merely do better than ignorance, but ignorance is known to be inferior to concealment (see Proposition 2(i)). On the other hand, we know from the discussion of Lemma 8 and Proposition 2 (iii) that the benefits of disclosure are small if a mitigation of the truth-telling problem is important, and this truth-telling effect becomes more severe if $\pi_{P}$ increases. ${ }^{16}$ This leads to an increasing relative profitability of concealment if $\pi_{P}$ grows, and suggests that concealment can be the optimal timing of information release if $I T_{P}$ is sufficiently uninformative or sufficiently informative.

\subsection{Superiority of Concealment?}

The sufficient conditions for superiority of disclosure in Proposition 3 suggest that concealment might be optimal for small values of $\pi_{P}$ (an uninformative $I T_{P}$ ), and for large values of $\pi_{P}$ if the truth-telling problem is pronounced. The following result proves this conjecture.

Proposition 4. (i) There is always an $\epsilon>0$ such that $\Phi^{C}>\Phi^{D}$ if $\pi_{P} \in(0, \epsilon)$. (ii) Suppose that $p_{0}>1 / 4$ or $p_{0}<1 / 4$ together with either $\pi_{P} \in\left(0, \frac{1-\sqrt{1-4 p_{0}}}{2\left(1-p_{0}\right)}\right)$ or $\pi_{P} \in\left(\frac{1+\sqrt{1-4 p_{0}}}{2\left(1-p_{0}\right)}, 1\right)$. Then $\Phi^{C}>\Phi^{D}$ if $\frac{e^{\prime}(\pi)}{e(\pi)}>\frac{1-\operatorname{Pr}\left(s_{P}=g\right)}{\frac{\operatorname{Pr}\left(s_{P}=g\right)}{1-p_{P}}-1} \forall \pi_{A}>0$.

Part (i) of the Proposition expresses that concealment is optimal whenever $I T_{P}$ is sufficiently uninformative. This builds on a familiar result from the literature on contracting under limited liability. Even if the stochastic signal is almost uncorrelated with the agent's performance ( $\pi_{P}$ close to zero) it remains a tool to reduce the agent's information rents.

\footnotetext{
${ }^{16}$ To see this just compare the terms proportional to $W(g, b)$ in Ineq. (10) with the terms proportional to $W(b, b)$ in Ineq. (12).
} 
The advantage of disclosure, however, disappears for $\pi_{P} \rightarrow 0$ as the agent is always hired and the implemented effort can not be adjusted.

Part (ii) complements Proposition 3. If the informativeness of $I T_{P}$ measured through $\pi_{P}$ is not within the range depicted in Proposition 3, Part (ii) shows that concealment is superior whenever the truth-telling problem is sufficiently pronounced. If the ratio $e^{\prime}\left(\pi_{A}\right) / e\left(\pi_{A}\right)$ is large for all $\pi_{A}$ it will in particular be large in equilibrium. Agency costs or costs of effort implementation $\left(e^{\prime}\left(\pi_{A}\right)\right)$ are then high compared to the pure cost reimbursement for the agent $\left(e\left(\pi_{A}\right)\right)$. But then, the profit difference between disclosure and concealment is mainly driven by the different costs of effort implementation and not by an adjusted hiring decision. Thus, concealment contracts reduce costs of effort implementation more effectively than disclosure contracts if $\pi_{P}$ is either very small or very large - in the former case because disclosure is not substantially better than ignorance, in the latter because truth-telling is easier to fulfill under concealment. Hence, in settings with substantial agency costs (i.e. $\frac{e^{\prime}\left(\pi_{A}\right)}{e\left(\pi_{A}\right)}$ large), and either low informativeness $\left(\pi_{P}\right.$ small) or a substantial truth-telling problem $\left(p_{0}\right.$ and $\pi_{P}$ large), concealment is superior.

\section{Discussion}

We have analyzed optimal contracts in a model where a principal can improve his knowledge about a project's quality by using two sources, one of them $\left(I T_{P}\right)$ without incentive problems, and the other one $\left(I T_{A}\right)$ burdened with moral hazard, adverse selection (truthtelling) and limited liability. We analyzed under which circumstances it is profit maximizing to offer a disclosure contract (IT $P$ is checked beforehand), or a concealment contract $\left(I T_{P}\right.$ is acquired after the agent reports his findings). We have found that a sufficient condition for the superiority of concealment is that the quality of $I T_{P}$ is sufficiently small. In this cases, $I T_{P}$ is almost worthless under a disclosure contract, while it can still be exploited to substantially reduce the agent's information rent under concealment. If $I T_{P}$ improves, it is more likely that disclosure is favorable as the possibility not to hire the agent and/or to adjust the agent's effort to the up-dated expected quality of the project becomes more important. However, increasing the informativeness of $I T_{P}$ also tightens the truth-telling constraint in a disclosure contract, so that concealment can well be superior because $I T_{P}$ is too informative for disclosure to perform well.

From a practical point of view, our findings first imply that the optimal timing depends crucially on the informativeness of $I T_{P}$. If, for instance, a bank has a relatively poor scoring system, it should run the automatic analysis only after the loan officer has submitted his report. Second, and maybe less intuitively, concealment can also prove superior if the automatic scoring system is sufficiently precise but it is very costly to make the agent work hard and to achieve truthful reporting. Both results suggest a closer look at the common practice of directly revealing automatic scoring results and subsequently contracting with a loan officer. In cases without a pronounced truth-telling problem (like in journalrefereeing, for instance), an immediate disclosure of existing information proves useful. The comparative statics with respect to $\pi_{P}$ as identified in this paper offers a guideline for 
optimal contract design. In particular, our contribution shows that a mechanism designer or principal does not necessarily benefit from new information if the agency problem at hand includes both moral hazard and adverse selection unless he offers a contract with a tailor-made timing of information acquisition.

Let us now discuss the robustness of our findings. In the loan officer-case, risk-aversion seems to be a natural extension. In the optimal concealment contract, the principal will then also pay positive wages if the signal and the report do not coincide, because this leads to better risk-sharing. Still, the detection premium would be higher if the signal is bad (to reduce the agent's rent). The basic trade-off analyzed in the paper would still exist with risk-aversion, but the usual trade-off between insurance and incentives would result in a less transparent exposition.

Moreover, the principal-agent relationship considered in our paper may be a repeated one. In an (infinitely) repeated game the following should be an equilibrium: with some (arbitrarily small) probability the principal invests even if the agent reports a bad signal, and threatens to end the contractual relationship if the project yields $R$ (which means that the agent has lied with probability one). This should almost eliminate the truth-telling problem, since the agent loses all of his future rents. However, investing even with a bad signal may not be renegotiation-proof, and we enter a discussion of commitment that is clearly beyond the objective of our model.

With respect to the information technologies $I T_{P}$ and $I T_{A}$ we made five crucial assumptions. First, both technologies $I T_{P}$ and $I T_{A}$ are such that a bad signal implies that the project certainly fails. While this assumption captures central features of some applications like auditing (for environmental protection, for instance, an expertise usually detects negligent firms - i.e. bad projects) or simple credit scoring (the system identifies nontrustworthy borrowers), it seems not very appealing for other fields like e.g. consultancy or scientific reviewing processes. In a companion working paper (Feess, Schieble, and Walzl (2004)), we assume that a good (bad) signal increases (decreases) the (ex-post) probability of a good project. This modelling obviously contains the model used in this paper as a special cases. While the superiority of concealment for almost uninformative signals can also be shown in such an environment, the identification of parameter regions that support the optimality of concealment for very informative signals and superiority of disclosure for intermediate technologies turns out to be much more cumbersome. In fact, we can only provide examples that support the same intuition as developed in this paper.

Second, effort costs for the agent are modelled such that the agent is always hired as long as the principal does not know that the project will fail (in particular after he received $s_{P}=b$ in a disclosure contract). One could conjecture a bias in favor of concealment due to this modelling detail. For certain effort costs $e(\pi), s_{P}=g$ could suggest direct investment without hiring the agent in a disclosure contract (resembling another advantage of disclosure not accounted for in our model). Clearly, this additional advantage would introduce another configuration that supports disclosure as the optimal timing option. If $\pi_{P}$ and therefore $p_{P}$ (the ex-post probability of a successful project) is close to one, disclosure will always be the optimal timing option, as $I T_{P}$ detects good projects (almost) with certainty (and without any costs), while the agent is still hired under concealment 
(and receives positive rents). While this does not question superiority of concealment for low values of $\pi_{P}$ it clearly qualifies our findings regarding very informative technologies. One can easily construct configurations where concealment is superior for $\pi_{P}$ sufficiently small $\left(\pi_{P}<\pi_{P}^{1} \in(0,1)\right.$, say) and in an intermediate region (between $\pi_{P}^{2} \in(0,1)$ and $\pi_{P}^{3} \in(0,1)$ with $\left.\pi_{P}^{2}<\pi_{P}^{3}\right)$, while disclosure is optimal for $\pi_{P} \in\left(\pi_{P}^{1}, \pi_{P}^{2}\right)$ and for any $\pi_{P}>\pi_{P}^{3}$. We discuss this in more detail in the companion working paper (Feess, Schieble, Walzl (2004)).

Third, we assumed $I T_{P}$ to be costless (instead of e.g. endowing the principal with a technology more comparable to $I T_{A}$ where he exerts effort $e\left(\pi_{P}\right)$ and thereby detects bad projects with probability $\pi_{P}$ ). In such a setting one would wish to identify characteristics of the principal's effort cost function that support the various timing options. In fact, our contribution offers several insights for such an environment. It is a corollary of Proposition 3(i) that concealment is superior if the principal's cost function is sufficiently steep. On the other hand, it is an implication of Proposition 3 and 4(ii) that concealment is not necessarily inferior if the principal's effort is sufficiently cheap (as high informativeness does not imply superiority of disclosure). In a similar way, our analysis suggests that the principal's optimal effort under concealment is not necessarily lower than under disclosure (which could be conjectured if disclosure was optimal as long as $I T_{P}$ would be sufficiently informative). Hence, while the crucial effects at work are identical to the dynamics described in this paper, the optimal timing and the principal's optimal effort level would depend on tiny details of the respective cost-functions that are not straightforward to identify.

Fourth, we followed the benchmark case of Ottaviani and Prat (2001) in assuming that the signal of $I T_{P}$ is (verifiable) public information once it has been checked by the principal. Our results possess a certain robustness in this respect. As the signal is binary it is easy to see that the principal can not improve upon the contracts discussed in this paper by (partially) hiding private information. A comparison of (non-verifiable) private and (verifiable) public information would therefore require a richer signal-space and a re-iteration of Ottaviani and Prat's (2001) analysis of combination of moral hazard and adverse selection. In such a case, also our restriction to pure strategies for the principal would no longer be an innocent one.

Fifth and related, none of the technologies requires the investment of fixed costs. Assuming that checking $I T_{P}$ asks for a fixed fee of $f_{P}$ and hiring the agent costs $f_{A}$ in advance, does not alter the effects identified in the paper but adds other driving forces that may well influence the optimal timing decision. Consider, for instance, a situation where checking $I T_{P}$ is quite costly (i.e. $f_{P}>>f_{A}$ ). While the principal has to invest into $I T_{P}$ in a disclosure contract, he can design a concealment contract where he commits himself to check only with a small positive probability. In such a situation, concealment will prove superior because of the respective rent-reduction effect. On the other hand, if hiring the agent requires substantial costs while checking $I T_{P}$ does not $\left(f_{A}>>f_{P}\right)$ disclosure can prove optimal as the agent is hired less often. In the presence of fixed costs the distinction between private and public information becomes also more pronounced as the principal has then an incentive to check $I T_{P}$ with a positive probability bounded away from 1 under concealment and disclosure. We consider it an interesting topic for further research to 
complement our insights about the nature of agency costs in the presence of moral hazard and adverse selection with Ottaviani and Prat (2001)'s findings on the relation between public and private information.

\section{Appendix 1: Proofs}

\section{Proof of Lemma 1}

If the principal does not hire the agent (part (i)), he will invest if and only if $s_{P}=g$ (part (ii)) as a bad signal implies that the project will yield zero profit. Following this strategy, the principal's profit is as given in part (iii), and $\frac{d \Phi^{0}}{d \pi_{P}}=\left(1-p_{0}\right) I>0$ (part (iv)).

\section{Proof of Lemma 2}

(i)-(iii). Obvious. (iv) From $\operatorname{Pr}\left(s_{P}=g\right)\left(1-p_{P}\right)=\left(1-p_{0}\right)\left(1-\pi_{P}\right)$ and the Envelope Theorem is follows that $\frac{d \Phi\left(C_{F B}\right)}{d \pi_{P}}=\left(1-p_{0}\right)\left(\left(1-\pi_{A}^{F}\right) I+e\left(\pi_{A}^{F}\right)\right)>0$.

\section{Proof of Lemma 3}

Using Eqn. (2), the principal's profit simplifies to $\Phi=p_{0} R-I+\left(1-p_{0}\right) \pi_{A} I-p_{0} W(g, g)-$ $\left(1-p_{0}\right) \pi_{A} W(g, b)-\pi_{A} e^{\prime}\left(\pi_{A}\right)$, which has to be maximized with respect to $W(b), W(g, g)$, and $W(g, b)$ subject to the limited liability constraints. $\Phi$ is monotone decreasing in $W(g, b)$

and $W(g, g)$. Hence, the (unique) solution is given by (i), and (ii) $\left(\widetilde{W}^{I}(b)=\frac{e^{\prime}\left(\widetilde{\pi}_{A}^{I}\right)}{1-p_{0}}\right.$ follows directly from Eqn. (2)). (iii) follows from $m=s_{A}$ and the characteristics of $I T_{A}$. (iv) is the principal's profit for an optimally chosen $\pi_{A}-$ see (i)

\section{Proof of Lemma 4}

(i)-(ii) follow from Lemma 3 by substituting $p_{P}$ for $p_{0}$ and re-specifying transfers for the case $s_{P}=g$. (iii) follows from the observability and characteristics of $I T_{P}$ and $I T_{A}$. (iv) holds due to Lemma 3 and (i)-(iii). (v) $\frac{d \Phi}{d \pi_{P}}=\left(1-p_{0}\right)\left(\left(1-\pi_{A}\right) I+\tilde{\pi}_{A}^{D} e^{\prime}\left(\tilde{\pi}_{A}^{D}\right)\right)>0$.

\section{Proof of Lemma 5}

Using Eqn. (4), the principal's profit simplifies to $\Phi=p_{0}(R-I-W(g, g, g))-(1-$ $\left.p_{0}\right)\left(1-\pi_{P}\right)\left(1-\pi_{A}\right) I-\pi_{A} e^{\prime}\left(\pi_{A}\right)-\left(1-p_{0}\right)\left(\left(1-\pi_{P}\right) W(g, g, b)+\pi_{P} W(b, g)\right)$, which has to be maximized with respect to $W(b, b), W(g, b), W(g, g, g), W(g, g, b)$, and $W(b, g)$ subject to the limited liability constraints. $\Phi$ is monotone decreasing in $W(g, g, g), W(g, g, b)$, and $W(b, g)$. Hence, all the respective limited liability constraints have to be binding and we are left with (ICC) that has to be fulfilled by an appropriate choice of $W(b, b)$ and $W(g, b)$ - see (ii). (i) is the first order condition for the optimal signal quality $\widetilde{\pi}_{A}^{C}$. (iii) follows 
once again from observability and signal characteristics. (iv) is obvious, and taking the derivative yields $(\mathrm{v}): \frac{d \widetilde{\Phi}^{C}}{d \pi_{P}}=\left(1-p_{0}\right)\left(1-\widetilde{\pi}_{A}^{C}\right) I>0$.

\section{Proof of Proposition 1}

(i) follows from a comparison of the first order conditions in Lemma 2, 3, 4, and 5 (i) and the assumptions on $e(\pi)$. (ii) $\Phi^{F}>\widetilde{\Phi}^{D}$ follows from $\pi^{F} \neq \widetilde{\pi}^{D}$. For $\widetilde{\Phi}^{D}>\widetilde{\Phi}^{C}$, suppose that the principal implements the (suboptimal) signal quality $\widetilde{\pi}_{A}^{C}$ in the disclosure contract. Let us denote the respective profit by $\widetilde{\Phi}^{D}\left(\pi_{A}=\widetilde{\pi}_{A}^{C}\right)$. Then $\widetilde{\Phi}^{D}\left(\pi_{A}=\widetilde{\pi}_{A}^{C}\right)-\widetilde{\Phi}^{C}=$ $\left(1-p_{0}\right) \pi_{P} \widetilde{\pi}_{A}^{C} e^{\prime}\left(\widetilde{\pi}_{A}^{C}\right)>0$. But as the principal's profit from the optimal disclosure contract has to be strictly higher than $\widetilde{\Phi}^{D}\left(\pi_{A}=\widetilde{\pi}_{A}^{C}\right), \widetilde{\Phi}^{D}>\widetilde{\Phi}^{C}$ follows. Suppose now that the principal implements the (suboptimal) signal quality $\widetilde{\pi}_{A}^{I}$ through a concealment contract. Then $\widetilde{\Phi}^{C}\left(\pi_{A}=\widetilde{\pi}_{A}^{I}\right)-\widetilde{\Phi}^{I} \geq 0$ implies $\widetilde{\Phi}^{C}>\widetilde{\Phi}^{I}$ as - once again - implemented efforts are nonidentical for the different contract types (see (i)) and profits for an optimal concealment contract are strictly higher than $\widetilde{\Phi}^{C}\left(\pi_{A}=\widetilde{\pi}_{A}^{C}\right)$.

\section{Proof of Lemma 6}

(i) Suppose not. This means that the principal's decision is independent of the agent's report. But then, hiring the agent has no benefit. (ii) If the agent lies, he either sends $m=g$ or $m=b$ regardless of his findings. Define $P(m)$ as his expected payment in this case. Then, his expected utility is $U\left(\pi_{A}\right)=P(m)-e\left(\pi_{A}\right)$ which is strictly decreasing in $\pi_{A}$.

\section{Proof of Lemma 7}

Inserting Eqn. (2) in the principal's profit function simplifies the program to maximize $\Phi=p_{0} R-I+\left(1-p_{0}\right) \pi_{A} I-p_{0} W(g, g)-\left(1-p_{0}\right) W(g, b)-\pi_{A} e^{\prime}\left(\pi_{A}\right)$ with respect to $W(b)$, $W(g, g)$, and $W(g, b)$ subject to the limited liability and truth-telling constraints. The corresponding Lagrangian is given by

$$
L=\Phi+\lambda_{T T}\left(U\left(\pi_{A}\right)-W(b)\right)+\lambda_{W(g, g)} W(g, g)+\lambda_{W(g, b)} W(g, b)+\lambda_{W(b)} W(b)
$$

and the system of first order conditions is

$$
\begin{aligned}
\frac{\partial L}{\partial W(g, g)} & =-p_{0}+\lambda_{T T} p_{0}+\lambda_{W(g, g)}=0 \\
\frac{\partial L}{\partial W(g, b)} & =-\left(1-p_{0}\right)+\frac{\partial \Phi}{\partial \pi_{A}} \frac{d \pi_{A}}{d W(g, b)}+\lambda_{T T}\left(1-p_{0}\right)\left(1-\pi_{A}\right)+\lambda_{W(g, b)}=0 \\
\frac{\partial L}{\partial W(b)} & =\frac{\partial \Phi}{\partial \pi_{A}} \frac{d \pi_{A}}{d W(b)}+\lambda_{T T}\left(\left(1-p_{0}\right) \pi_{A}-1\right)+\lambda_{W(b)}=0
\end{aligned}
$$


where $\frac{\partial \Phi}{\partial \pi_{A}}=-\pi_{A} e^{\prime \prime}\left(\pi_{A}\right)-e^{\prime}\left(\pi_{A}\right)+\left(1-p_{0}\right) I, \frac{d \pi_{A}}{d W(g, b)}=-\frac{1-p_{0}}{e^{\prime \prime}\left(\pi_{A}\right)}$, and $\frac{d \pi_{A}}{d W(b)}=\frac{1-p_{0}}{e^{\prime \prime}\left(\pi_{A}\right)}$ (the last two results are applications of the Implicit Function Theorem to Eqn. (2)). To solve System (13) observe first that $\lambda_{W(g, b)}=0$ (a necessary requirement for $W(g, b)>0$ ) implies that $\lambda_{T T}>0$ is fully determined by $\frac{\partial L}{\partial W(g, b)}=0$. This implies $\lambda_{W(b)}>0$ as otherwise $\frac{\partial L}{\partial W(b)}=0$ could not be fulfilled. But then $W(b)=0$ such that the incentive compatibility constraint can not be fulfilled for a positive signal quality. Therefore, we will assume $W^{I}(g, b)=0$ from now on, and suppose that $\frac{\partial L}{\partial W(g, b)}=0$ is solved by an appropriate choice of $\lambda_{W(g, b)}>0$. Now suppose that $W(g, g)=0$. In this case the truth-telling constraint Ineq. (6) can not be satisfied. But if $W(g, g)>0$ (and thus $\lambda_{W(g, g)}=0$ ), the first equation in System (13) implies that $\lambda_{T T}=1$. Hence, the truth-telling constraint is binding and $W^{I}(g, g)$ is given as in (ii). Plugging $\lambda_{T T}=1$ and $\lambda_{W(b)}=0$ into $\frac{\partial L}{\partial W(b)}=0$ yields the effort selection constraint $\left(1-p_{0}\right) I=\frac{e^{\prime \prime}\left(\pi_{A}\right)}{1-p_{0}}+e^{\prime}\left(\pi_{A}\right)$ (as depicted in (i)). (iii) follows from the satisfied truth-telling constraints and the nature of the signal. Finally, we get (iv) by inserting $W(g, b)=0, W^{I}(b)$, and $W^{I}(g, g)$ into $\Phi$.

\section{Proof of Lemma 8}

The proof of (i) - (iv) is identical to the proof of Lemma 7 except that $p_{0}$ is substituted by $p_{P}$, and that transfers account for $s_{P}=g$ whenever the agent is hired. (v) and (vi) follows directly from (iv). (vii) is shown by the example in Appendix 2.

\section{Proof of Lemma 9}

Inserting Eqn. (4) in the principal's profit function simplifies the program to maximize $\Phi=p_{0}(R-I-W(g, g, g))-\pi_{A} e^{\prime}\left(\pi_{A}\right)-\left(1-p_{0}\right)\left(\left(1-\pi_{P}\right) W(g, g, b)+\pi_{P} W(b, g)\right)-\left(1-p_{0}\right)(1-$ $\left.\pi_{P}\right)\left(1-\pi_{A}\right) I$ with respect to $W(b, b), W(g, b), W(b, g) W(g, g, g)$, and $W(g, g, b)$ subject to the limited liability constraint and the truth-telling constraint given by Ineq.(12). The corresponding Lagrangian is

$$
\begin{aligned}
L= & \Phi+\lambda_{T T}\left(U\left(\pi_{A}\right)-p_{0} W(g, b)-\left(1-p_{0}\right)\left(\pi_{P} W(b, b)+\left(1-\pi_{P}\right) W(g, b)\right)\right) \\
& +\lambda_{W(g, g, g)} W(g, g, g)+\lambda_{W(g, g, b)} W(g, g, b) \\
& +\lambda_{W(g, b)} W(g, b)+\lambda_{W(b, b)} W(b, b)+\lambda_{W(b, g)} W(b, g)
\end{aligned}
$$

and the system of first order conditions is 


$$
\begin{aligned}
\frac{\partial L}{\partial W(g, g, g)}= & -p_{0}+\lambda_{T T} p_{0}+\lambda_{W(g, g, g)}=0 \\
\frac{\partial L}{\partial W(g, g, b)}= & -\left(1-p_{0}\right)\left(1-\pi_{P}\right)+\frac{\partial \Phi}{\partial \pi_{A}} \frac{d \pi_{A}}{d W(g, g, b)} \\
& +\lambda_{T T}\left(1-p_{0}\right)\left(1-\pi_{P}\right)\left(1-\pi_{A}\right)+\lambda_{W(g, g, b)}=0 \\
\frac{\partial L}{\partial W(b, g)}= & -\left(1-p_{0}\right) \pi_{P}+\frac{\partial \Phi}{\partial \pi_{A}} \frac{d \pi_{A}}{d W(b, g)} \\
& +\lambda_{T T}\left(1-p_{0}\right) \pi_{P}\left(1-\pi_{A}\right)+\lambda_{W(g, g, b)}=0 \\
\frac{\partial L}{\partial W(g, b)}= & \frac{\partial \Phi}{\partial \pi_{A}} \frac{d \pi_{A}}{d W(g, b)} \\
& +\lambda_{T T}\left(\left(1-p_{0}\right)\left(1-\pi_{P}\right)\left(\pi_{A}-1\right)-p_{0}\right)+\lambda_{W(g, b)}=0 \\
\frac{\partial L}{\partial W(b, b)}= & \frac{\partial \Phi}{\partial \pi_{A}} \frac{d \pi_{A}}{d W(b, b)}+\lambda_{T T}\left(1-p_{0}\right) \pi_{P}\left(\pi_{A}-1\right)+\lambda_{W(b, b)}=0
\end{aligned}
$$

where $\frac{\partial \Phi}{\partial \pi_{A}}=\left(-\pi_{A} e^{\prime \prime}\left(\pi_{A}\right)-e^{\prime}\left(\pi_{A}\right)+\left(1-p_{0}\right)\left(1-\pi_{P}\right) I\right), \frac{d \pi_{A}}{d W(g, g, b)}=-\frac{\left(1-p_{0}\right)\left(1-\pi_{P}\right)}{e^{\prime \prime}\left(\pi_{A}\right)}, \frac{d \pi_{A}}{d W(b, g)}=$ $-\frac{\left(1-p_{0}\right) \pi_{P}}{e^{\prime \prime}\left(\pi_{A}\right)}, \frac{d \pi_{A}}{d W(b, b)}=\frac{\left(1-p_{0}\right) \pi_{P}}{e^{\prime \prime}\left(\pi_{A}\right)}$ and $\frac{d \pi_{A}}{d W(g, b)}=\frac{\left(1-p_{0}\right)\left(1-\pi_{P}\right)}{e^{\prime \prime}\left(\pi_{A}\right)}$.

Suppose now that either $W(b, g)$ or $W(g, g, b)$ are strictly positive (and the corresponding Lagrange multiplier is thus zero). Then $\lambda_{T T}$ is fully determined by $\left(\frac{\partial L}{\partial W(b, g)}=0\right)$ or $\left(\frac{\partial L}{\partial W(g, g, b)}=0\right)$, respectively. Inserting such a $\lambda_{T T}$ into $\frac{\partial L}{\partial W(b, b)}$ and $\frac{\partial L}{\partial W(g, b)}$ implies $\lambda_{W(b, b)}>0$ (i.e. $W(b, b)=0$ ) and $\lambda_{W(g, b)}>0$ (i.e. $W(g, b)=0$ ) - which violates (ICC). Hence, we get $W^{C}(g, g, b)=W^{C}(b, g)=0$. This given, $W(g, g, g)$ has to be strictly positive to satisfy Ineq. (12) which means that $\lambda_{W(g, g, g)}=0$ such that $\frac{\partial L}{\partial W(g, g, g)}=0$ implies $\lambda_{T T}=1$ (i.e. the truth-telling constraint is binding). Inserting $\lambda_{T T}=1$ into $\frac{\partial L}{\partial W(g, b)}=0$ and $\frac{\partial L}{\partial W(b, b)}=0$ shows that both equations can be solved simultaneously for weakly positive Lagrange multipliers if and only if $\lambda_{W(g, b)}>0$ (i.e. $\left.W(g, b)=0\right)$ and $\lambda_{W(b, b)}=0$ with (ICC) $e^{\prime \prime}\left(\pi_{A}\right)+e^{\prime}\left(\pi_{A}\right)=\left(1-p_{0}\right)\left(1-\pi_{P}\right) I$ (see $\left.(\mathrm{i})\right) . W(b, b)>0$ is therefore determined by incentive compatibility in Eqn. (4) (see (ii)). (iii) follows from the satisfied truth-telling constraints and the nature of the signal. (iv) follows from inserting the transfers as specified in (ii) into the principal's profit function, and this yields $(\mathrm{v}) \frac{d \Phi}{d \pi_{P}}=\left(1-p_{0}\right)\left(1-\pi_{A}\right)>0$.

\section{Proof of Lemma 10}

(i) and (ii). First observe that $\Phi^{0}=\left.\Phi^{D}\right|_{\pi_{A}=0}=\left.\Phi^{C}\right|_{\pi_{A}=0}$. But as Lemma 8 (i) and 9(i) and the assumptions on $e(\pi)$ imply that $\pi_{A}^{D}>0$ and $\pi_{A}^{C}>0$ it follows that $\Phi^{0}<\Phi^{D}$ and $\Phi^{0}<\Phi^{C}$. 


\section{Proof of Proposition 2}

(i) $\Phi^{C}-\Phi^{I}=\left(1-p_{0}\right)\left(\pi_{A}^{C}-\pi_{A}^{I}\right) I+\left(1-p_{0}\right)\left(1-\pi_{A}^{C}\right) \pi_{P} I-e^{\prime}\left(\pi_{A}^{C}\right)+\frac{e^{\prime}\left(\pi_{A}^{I}\right)}{1-p_{0}}-e\left(\pi_{A}^{C}\right)+$ $e\left(\pi_{A}^{I}\right)$. Now suppose that in the concealment contract the suboptimal signal quality $\pi_{A}^{I}$ is implemented. This leads to $\Phi^{C}-\Phi^{I}>\left(1-p_{0}\right)\left(1-\pi_{A}^{I}\right) \pi_{P} I+e^{\prime}\left(\pi_{A}^{I}\right)\left(\frac{1}{1-p_{0}}-1\right)>0$.

(ii) First, $\Phi^{D}$ is continuous in $\pi_{P}$ and $\lim _{\pi_{P} \rightarrow 0} \Phi^{D}=\Phi^{I}$. Second, $\frac{d \Phi^{D}}{d \pi_{P}}=\left(1-p_{0}\right)(1-$ $\left.\pi_{A}^{D}\right) I+e^{\prime}\left(\pi_{A}^{D}\right) \frac{\left(1-p_{0}\right)\left(1-2 p_{P}\right)}{\left(1-p_{P}\right)^{2}}+\left(1-p_{0}\right) e\left(\pi_{A}^{D}\right)$ such that $p_{P} \leq 1 / 2$ is sufficient condition for $\frac{d \Phi^{D}}{d \pi_{P}}>0$ (see Lemma $8(\mathrm{vi})$ ). And as $\frac{d \Phi^{I}}{d \pi_{P}}=0$, (ii) follows. (iii) follows in a similar way from $\lim _{\pi_{P} \rightarrow 0} \Phi^{D}=\Phi^{I}$ and Lemma 8 (vii).

\section{Proof of Proposition 3}

$\Phi^{C}>\Phi^{I}$ has already been proven by Proposition 2 (i). To show that $\Phi^{D}>\Phi^{C}$ holds, consider the difference $\Phi^{D}-\Phi^{C}$ and suppose that (the suboptimal signal quality) $\pi_{A}^{C}$ is implemented in the disclosure contract and denote the respective profit by $\Phi^{D}\left(\pi_{A}=\pi_{A}^{C}\right)$. We get $\Phi^{D}\left(\pi_{A}=\pi_{A}^{C}\right)-\Phi^{C}=e^{\prime}\left(\pi_{A}^{C}\right) \frac{1-p_{P}-\operatorname{Pr}\left(s_{P}=g\right)}{1-p_{P}}+e\left(\pi_{A}^{C}\right)\left(1-\operatorname{Pr}\left(s_{P}=g\right)\right)$. A sufficient condition for this expression to be positive is $\frac{1-p_{P}-\operatorname{Pr}\left(s_{P}=g\right)}{1-p_{P}}>0$ which holds whenever $\pi_{P} \in\left[\frac{1-\sqrt{1-4 p_{0}}}{2\left(1-p_{0}\right)}, \frac{1+\sqrt{1-4 p_{0}}}{2\left(1-p_{0}\right)}\right]$. For this interval to be a non-empty, non-degenerate, connected subset of $(0,1), p_{0}$ has to be smaller than $1 / 4$ - for $p_{0}=1 / 4$ the condition shrinks to $\pi_{P}=2 / 3$ and in the limit $p_{0} \rightarrow 0$ the interval becomes $(0,1)$

\section{Proof of Proposition 4}

(i) This follows directly from $\Phi^{C}>\Phi^{I}$ (see Proposition 2(i)), the continuity of $\Phi^{D}$ in $\pi_{P}$, and $\lim _{\pi_{P} \rightarrow 0} \Phi^{D}=\Phi^{I}$ as shown in the proof of Proposition 2(ii). (ii) Consider the difference $\Phi^{C}-\Phi^{D}$ and suppose that (the suboptimal signal quality) $\pi_{A}^{D}$ is implemented in the concealment contract. We denote the respective profit by $\Phi^{C}\left(\pi_{A}=\pi_{A}^{D}\right)$ and get $\Phi^{C}\left(\pi_{A}=\pi_{A}^{D}\right)-\Phi^{D}=-e^{\prime}\left(\pi_{A}^{D}\right) \frac{1-p_{P}-P r\left(s_{P}=g\right)}{1-p_{P}}-e\left(\pi_{A}^{D}\right)\left(1-\operatorname{Pr}\left(s_{P}=g\right)\right)$. A sufficient condition for the first term to be positive (i.e. $\frac{1-p_{P}-\operatorname{Pr}\left(s_{P}=g\right)}{1-p_{P}}<0$ ) is that $p_{0}>1 / 4$ or $p_{0} \leq 11 / 4$ together with $\pi_{P} \in\left(0, \frac{1-\sqrt{1-4 p_{0}}}{2\left(1-p_{0}\right)}\right)$ or $\pi_{P} \in\left(\frac{1+\sqrt{1-4 p_{0}}}{2\left(1-p_{0}\right)}, 1\right)$ - see also the proof of Proposition 3. Now suppose that $\frac{1-p_{P}-\operatorname{Pr}\left(s_{P}=g\right)}{1-p_{P}}<0$. Then $\Phi^{C}\left(\pi_{A}=\pi_{A}^{D}\right)-\Phi^{D}>0$ whenever $\frac{e^{\prime}\left(\pi_{A}\right)}{e\left(\pi_{A}\right)}>$ $\frac{\left(1-\operatorname{Pr}\left(s_{P}=g\right)\right)\left(1-p_{P}\right)}{\operatorname{Pr}\left(s_{P}=g\right)-\left(1-p_{P}\right)}$ for all $\pi_{A} \in(0,1) .{ }^{17}$

\footnotetext{
${ }^{17}$ Note that the right-hand-side of the last inequality is positive and finite in the given range of $\pi_{P}$. E.g. $e\left(\pi_{A}\right)=\pi_{A}^{n}+\delta\left(-\ln \left(1-\pi_{A}\right)-\pi_{A}\right)$ can satisfy this condition for all $\pi_{A}$, i.e. there is a $\widetilde{\delta}>0$ and $\widetilde{n}>0$ such that $\frac{e^{\prime}\left(\pi_{A}\right)}{e\left(\pi_{A}\right)}>\frac{\left(1-\operatorname{Pr}\left(s_{P}=g\right)\right)\left(1-p_{P}\right)}{\operatorname{Pr}\left(s_{P}=g\right)-\left(1-p_{P}\right)} \forall \pi_{A} \in(0,1)$ if $\delta \in(0, \widetilde{\delta})$ and $n>\widetilde{n}$.
} 


\section{Appendix 2: Example for $\frac{d \Phi^{D}}{d \pi_{P}}<0$}

Suppose that $e\left(\pi_{A}\right)=C I \frac{1}{2} \pi_{A}^{2}+\delta\left(-\ln \left(1-\pi_{A}\right)-\pi_{A}\right)$. As long as $\delta>0$, this effort function satisfies all our assumptions ${ }^{18}$, while for $\delta>0$ sufficiently small and $\pi_{A} \in[0,1)$, the optimization program can be restricted to $e\left(\pi_{i}\right)=C I \frac{1}{2} \pi_{A}^{2}$ and an analysis of the boundary for $\pi_{A} \in[0,1)$. Then, the first order conditions for a disclosure contract is

$$
\begin{aligned}
\left(1-p_{P}\right) I & =\frac{e^{\prime \prime}\left(\pi_{A}\right)}{1-p_{P}}+e^{\prime}\left(\pi_{A}\right) \\
& =\frac{C I}{1-p_{P}}+C I \pi_{A}
\end{aligned}
$$

such that

$$
\pi_{A}^{D}=\frac{1-p_{P}}{C}-\frac{1}{1-p_{P}}
$$

We restrict ourselves to parameter configurations where the agent is hired $\left(\pi_{A}>0\right)$ and where $C I \frac{1}{2} \pi^{2}$ is indeed an approximation of $e\left(\pi_{A}\right)\left(\pi_{A}<1\right)$. This requires $C \in\left(C_{0}, C_{1}\right)$ with $C_{0}=\frac{\left(1-p_{P}\right)^{2}}{2-p_{P}}$ and $C_{1}=\left(1-p_{P}\right)^{2}$. We use this specification of $e(\pi)$ to give an example for a profit function in an optimal disclosure contract that is locally decreasing in $\pi_{P}$ at $\pi_{P}=0$. Observe first that

$$
\left.\frac{d \Phi^{D}}{d \pi_{P}}\right|_{\pi_{P}=0}=\left(1-p_{0}\right)\left(1-\pi_{A}^{D}\right) I-e^{\prime}\left(\pi_{A}^{D}\right) \frac{1-2 p_{0}}{1-p_{0}}+\left(1-p_{0}\right) e\left(\pi_{A}^{D}\right) .
$$

Inserting $e(\pi)$ as specified above and $\pi_{A}^{D}$ as in Eqn. (15) yields $\left.\frac{d \Phi^{D}}{d \pi_{P}}\right|_{\pi_{P}=0}=-I\left(-4 p_{0}^{2}+\right.$ $\left.3 C p_{0}+6 p_{0}^{3}-4 p_{0}^{4}+p_{0}^{5}-3 C p_{0}^{2}+p_{0}^{3} C-C^{2} p_{0}+p_{0}-C\right) /\left(C\left(1-p_{0}\right)^{2}\right)$ which is negative for every $C \in\left(1 / 2\left(-1+p_{0}-\sqrt{1-2 p_{0}+5 p_{0}^{2}}\right)\left(1-p_{0}\right)^{2} / p_{0}, 1 / 2\left(-1+p_{0}+\sqrt{1-2 p_{0}+5 p_{0}^{2}}\right)\left(1-p_{0}\right)^{2} / p_{0}\right)$. Now note that the lower bound of this interval is negative for all $p_{0} \in(0,1)$ such that $\left.\frac{d\left(\Phi^{D}\right)}{d \pi_{P}}\right|_{\pi_{P}=0}<0$ whenever $C \in\left(C_{0}, C_{1}\right)$ and $C<1 / 2\left(-1+p_{0}+\sqrt{1-2 p_{0}+5 p_{0}^{2}}\right)(1-$ $\left.\left.p_{0}\right)^{2} / p_{0}\right) \equiv \widetilde{C}$. A sufficient condition for such a $C$ to exist is $p_{0}>3 / 4$. Then $C_{0}<\widetilde{C}<C_{1}$ and $\left.\frac{d\left(\Phi^{D}\right)}{d \pi_{P}}\right|_{\pi_{P}=0}<0$ whenever $C \in\left(C_{0}, \widetilde{C}\right)$.

\footnotetext{
${ }^{18}$ Only $e^{\prime \prime}(0)=0$ is not satisfied as this would drive the computation more cumbersome (solving cubic instead of quadratic equations). Instead we restrict ourselves to parameter regions where the agent is hired - see below.
} 


\section{References}

[1] Beaudry, P. (1994): Why an Informed Principal May Leave Rents to an Agent, International Economic Review, 35, 4, 821-832.

[2] Chade, H. and Silvers, R. (2002): Informed Principals, Moral Hazard, and the Value of a More Informative Technology, Economics Letters, 74, 291-300.

[3] Cremer, J. and P. McLean (1985): Optimal Selling Strategies under Uncertainty for a Discriminating Monopolist when Demands are Interdependent, Econometrica, 53, 354-362.

[4] Demski, J. and D. Sappington (1987a): Delegated Expertise, Journal of Accounting Research, 25 , 68-89.

[5] Demski, J. and D. Sappington (1987b): On the Timing of Information Release, Information Economics and Policy, 2, 307-316.

[6] Feess, E. and Walzl, M. (2004): When Sould Principals Acquire verifiable Information?, METEOR Research Memorandum 049/04.

[7] Gromb, D. and D. Martimort (2004): The Organization of Delegated Expertise, IDEI working paper No. 284.

[8] Lewis, T.R. and D. Sappington (1997): Information Management in Incentive Problems, Journal of Political Economy, 105, 4, 796-821.

[9] Maskin, E. and J. Tirole (1990): The Principal-Agent Relationship with an Informed Principal: The Case of Private Values, Econometrica, 58, 379-409.

[10] Maskin, E. and J. Tirole (1992): The Principal-Agent Relationship with an Informed Principal, II: Common Values, Econometrica, 60, 1-42.

[11] Milgrom, P. and R. Weber (1982): A Theory of Auctions and Competitive Biddig, Econometrica, 50, 1089-1122.

[12] Ottaviani, M. and A. Prat (2001): The Value of Public Information in Monopoly, Econometrica, 69, 1673-1683. 